This item was submitted to Loughborough's Research Repository by the author.

Items in Figshare are protected by copyright, with all rights reserved, unless otherwise indicated.

Improved high-frequency carrier voltage measurement for position estimation of switched-flux permanent magnet machines

PLEASE CITE THE PUBLISHED VERSION

http://dx.doi.org/10.1109/SLED.2015.7339268

PUBLISHER

(C) IEEE

VERSION

AM (Accepted Manuscript)

LICENCE

CC BY-NC-ND 4.0

REPOSITORY RECORD

Lin, Tzu-Chi, Zi-Qiang Zhu, and Kan Liu. 2019. "Improved High-frequency Carrier Voltage Measurement for Position Estimation of Switched-flux Permanent Magnet Machines". figshare.

https://hdl.handle.net/2134/24444. 


\title{
Improved High-Frequency Carrier Voltage Measurement for Position Estimation of Switched-Flux Permanent Magnet Machines
}

\author{
T.C. Lin, Z.Q. Zhu, and K. Liu
}

\begin{abstract}
The conventional 12/10 stator/rotor poles switched-flux permanent magnet (SFPM) machine is usually based on all poles wound topology, each phase comprising four winding coils in series connection. However, alternate coils of the same phase have different machine saliency characteristics. Moreover, in order to measure the high-frequency (HF) carrier voltage, the mid-tapered winding wires can be utilized. Consequently, the machine saliencies can be measured separately from two parts of winding coil connections. This paper investigates the influences of machine saliencies on the sensorless rotor position estimations based on different sequence of winding coil connections, in which the primary saliency may contain some additional harmonics referring to the secondary saliency that will degrade the overall sensorless control operations. Furthermore, a simple compensation method is proposed to reduce the influence of multiple saliencies to achieve more accurate sensorless rotor position estimation. By comparing with rotor positon estimations without the proposed compensation and $\mathrm{HF}$ carrier current based method, the effectiveness of improved sensorless rotor position estimation has been demonstrated experimentally, as well as the application to dual 3-phase SFPM machines.
\end{abstract}

Index Terms - high-frequency injection, machine saliency, machine winding configuration, permanent magnet synchronous machine (PMSM), switched-flux machine.

\section{INTRODUCTION}

High-frequency (HF) signal injection based sensorless control methods have become more and more popular for permanent magnet (PM) machines due to their good performance at zero and low speeds [1]-[3]. In terms of implementing HF injection based techniques, the machine saliency, mainly induced by the inductance variation, is an important requirement. Although the carrier signal injection based techniques are simple in principle, the non-ideality of machines and drives, including cross-saturation [4][5] and multiple saliencies [2], and inverter non-linearity [6], has apparent influence on the sensorless control operations. In [4], the existence of multiple saliencies (or a saliency with multiple harmonics) were fully investigated and the accuracy of rotor positon was affected by the additional saliency components.

In this paper, the basic theory of evaluating machine saliency using the HF injection technique is briefly described first. In

T.C. Lin and Z.Q. Zhu are with Department of Electronic and Electrical Engineering, University of Sheffield, Sheffield, U.K. (Tel: +44(0)1142225360, Fax: +44(0)1142225196, e-mail: T.C.Lin@sheffield.ac.uk and Z.Q.Zhu@sheffield.ac.uk) order to investigate the saliency level based on different sequence of winding coil connections (WCCs) in all poles wound switched-flux permanent magnet (SFPM) machines as will be introduced in section II, a simple measurement method is utilized. By injecting a HF carrier signal into the virtual synchronous reference frame while the rotor rotates at low speed, the carrier voltage response containing the full information of machine saliency can be obtained without any knowledge of the machine parameters. Further, by utilizing the mid-tapered winding wires, the machine saliency level can be measured separately as two sets of machine saliencies, in which the machine saliencies are similar to the machine based on the alternate poles wound machine. However, the secondary saliency may exist due to the additional harmonics in the carrier voltage signal. Hence, a very simple method is proposed to improve the sensorless control performance by cancelling estimation errors from two sets of windings. Furthermore, the effectiveness of sensorless rotor position estimation based on different sequence of WCCs will be demonstrated comparing with that of the sensorless control performance without compensation as well as the dual three-phase application.

\section{PROTOTYPE MACHINE TOPOLOGY AND TAPERED WINDING MEASUREMENT}

The conventional 12/10 stator/rotor poles SFPM machine, as shown in Fig. 1, is investigated in this paper. It has a very simple machine structure consisting of a robust rotor and PMs located in the stator.

The conventional structure of SFPM machine, Fig. 1 (a), is usually all poles wound as double layer machine, and each phase contains four winding coils in series connection [7][87]. The first sequence type of WCC is 1-4-7-10 which are in series connection to form one phase (Type-1), Fig. 2 (a), i.e. A1-A2-A3-A4 for phase A, and similarly for phases B and C, as shown in Figs. 1 (b). Two winding coils of two phases are placed in each stator slot. Differing from the Type-1, the second sequence of WCC for SFPM machine can also be re-arranged as the winding coils 1-7-4-10 are connected in series to operate as the phase A (Type-2), Fig. 2 (b), i.e. A1-A3-A2-A4 for phase $A$ and similarly for phases $B$ and $C$, as can be seen in Fig. 1 (b). In addition, it is also possible to control the prototype SFPM machines with dual three-phase inverters. In this case, the two three-phase windings by connecting winding coils 1 and 7 as $\mathrm{A} 1$ and $\mathrm{A} 2$ for set 1 , whilst winding coils 4 and 10 as $\mathrm{A} 1^{*}$ and $\mathrm{A} 2^{*}$ for set 2, Fig. 1 (c). 
More importantly, it should be noted that there is a difference in relative position between the machine stator poles and rotor poles. As can be seen from Fig. 1, the machine stator poles 1 and 7 are aligned with the rotor slots, whilst the machine stator poles 4 and 10 are faced the rotor poles. Due to different alignment between machine stator and rotor, the alternate coils in SFPM machine will exhibit different saliency features although the overall saliency in Type- 1 and Type- 2 WCCs is the same.

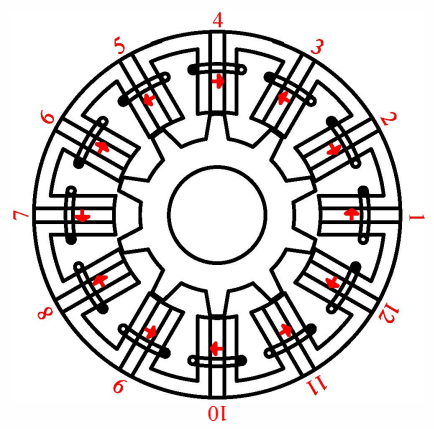

(a) Coil numbers

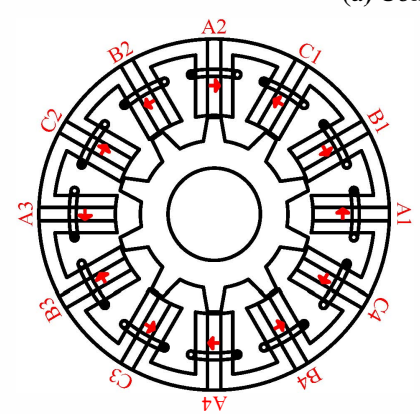

(b) All poles wound, single three-phase

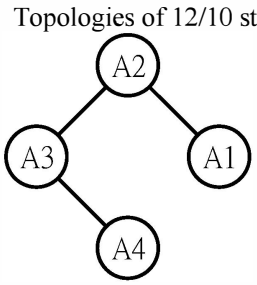

(a) Winding coil connection Type-1

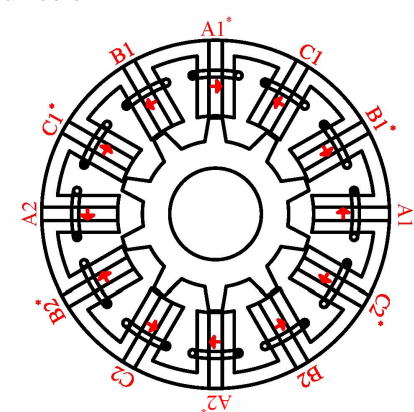

(c) All poles wound, dual three-phase
Fig. 1. Topologies of 12/10 stator/rotor SFPM machines

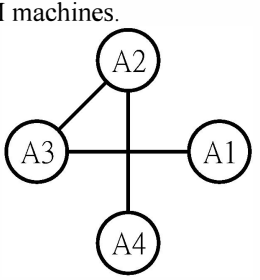

(d) Winding coil connection Type-2
Fig. 2. Sequence of winding coil connections.

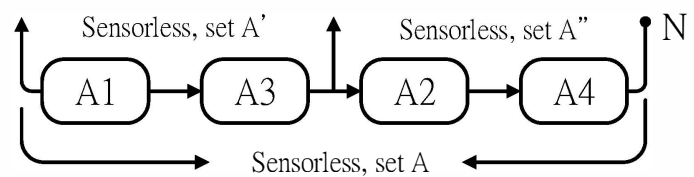

Fig. 3. Measurement of HF carrier voltage signals.

Additionally, with the aid of mid-tapered winding wires between winding coils A3 and A2 (Type-2), the machine saliency can be measured from the different part of the winding connections, i.e. winding coils A1 and A3 are referred to set A', and similarly winding coils A2 and A4 are presented as set A" as shown in Fig. 3. By connecting set $A^{\prime}$ and set A", the additional harmonics would be cancelled due to the coil compensation effect [9] which can be referred to set A, Fig. 3. Compared with Type-2, the saliency effect in Type-1 would be expected to be reduced due to saliency cancelling effect and the multiple saliencies will not exist. According to Type-2, the machine saliency measured from set $A^{\prime}$ is expected to be identical to that of set A" (Fig. 3), but each may exhibit the secondary saliency, which will affect the sensorless control performance.

\section{INVESTIGATION OF SALIENCY}

\section{A. High-Frequency Model of PM Machine}

In terms of machine saliency evaluation, the saliency level will be investigated firstly based on all poles wound machine in the different sequence of WCCs, which are shown in Figs. 2 and 3. Moreover, the basic machine saliency investigation will be firstly based on Type- 1 and Type- 2 for explanation. Then, the comparison of machine saliency level measured from the different part of WCC shown in Fig. 3 will be introduced afterword. For the PM machine with salient poles, the $d$-axis inductance is usually smaller than the q-axis inductance. Hence, it is possible to utilize the HF injection technique for the sensorless control operation. Then, the HF voltage model for a PM machine in the synchronous $d$ - and $q$ - axis reference frame can be expressed as

$$
\left[\begin{array}{l}
u_{d h} \\
u_{\mathbf{q} h}
\end{array}\right]=\left[\begin{array}{cc}
L_{d h} & 0 \\
0 & L_{\mathbf{q} h}
\end{array}\right] \cdot p\left[\begin{array}{l}
u_{d h} \\
u_{\mathbf{q} h}
\end{array}\right]
$$

where $u_{d h}, u_{q h}, i_{d h}$ and $i_{q h}$ are the $d$ - and $q$-axis HF injection voltage and HF carrier current, respectively. $L_{d h}$ and $L_{\boldsymbol{q} h}$ represent the $d$ - and $q$-axis HF inductances. When the cross-coupling between $d$ - and $q$-axis is considered, the HF voltage model according to (1) can be re-expressed as

$$
\left[\begin{array}{l}
u_{d h} \\
u_{\mathbf{q} h}
\end{array}\right]=\left[\begin{array}{cc}
L_{d h} & L_{d \boldsymbol{q} h} \\
L_{\mathbf{q} d h} & L_{\mathbf{q} h}
\end{array}\right] \cdot p\left[\begin{array}{l}
u_{d h} \\
u_{\mathbf{q} h}
\end{array}\right]
$$

where $L_{d h}, L_{q h}, L_{d q h}$ and $L_{q d h}$ are referred to the incremental $d$ and $q$-axis self and mutual inductances, respectively. Then, these HF inductances can be defined as

$$
\begin{aligned}
& L_{d h}=\frac{\partial \psi_{d}\left(i_{d}+i_{d h}, i_{\boldsymbol{q}}, \lambda_{m}\right)}{\partial i_{d}}, L_{\mathbf{q} h}=\frac{\partial \psi_{\boldsymbol{q}}\left(i_{d}+i_{\boldsymbol{q}}, i_{\boldsymbol{q} h}, \lambda_{m}\right)}{\partial i_{\boldsymbol{q}}} \\
& L_{d \boldsymbol{q} h}=\frac{\partial \psi_{d}\left(i_{d}+i_{\boldsymbol{q}}, i_{\boldsymbol{q} h}, \lambda_{m}\right)}{\partial i_{\boldsymbol{q}}}, L_{\mathbf{q} h}=\frac{\partial \psi_{\boldsymbol{q}}\left(i_{d}+i_{d h}, i_{\boldsymbol{q}}, \lambda_{m}\right)}{\partial i_{d}}
\end{aligned}
$$

In general, $L_{d q h}$ and $L_{q d h}$ are different and related to the influence of cross-saturation. Moreover, $L_{d h}$ and $L_{q h}$ are also different because of the rotor geometric and magnetic saturations. The difference between $L_{d h}$ and $L_{\boldsymbol{q} h}$ which is $L_{\mathbf{q} h}-L_{d h}$ indicates the saliency level of machine. Furthermore, if $L_{a h} L_{d h}$ is constant and large enough, the machine would be suitable for the method based on HF carrier signal injection. Hence, the saliency level has to be investigated first and a simple experimental investigation is utilized in the next section.

\section{B. Saliency Investigation}

To obtain the machine saliency information, a virtual synchronous reference frame is developed from the actual synchronous reference frame as shown in Fig. 4. Then, the transformation matrix $T(\Delta \theta)$ can be expressed as

$$
T(\Delta \theta)=\left[\begin{array}{cc}
\cos (\Delta \theta) & -\sin (\Delta \theta) \\
\sin (\Delta \theta) & \cos (\Delta \theta)
\end{array}\right]
$$


where $\Delta \theta=\theta_{r}^{e}-\theta_{r}$, which is the electrical rotor position estimation error. $\theta_{r}^{e}$ presents the estimated rotor position and $\theta_{r}$ is referred to the actual rotor position.

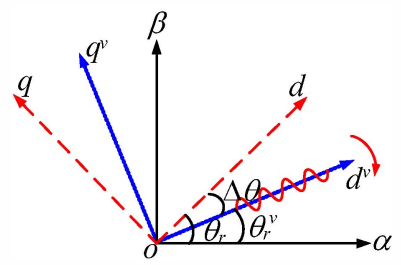

Fig. 4. Actual and virtual synchronous reference frames.

According to Fig. 4, the HF voltage model in virtual synchronous reference frame from the actual reference frame can be derived as

$$
\begin{aligned}
{\left[\begin{array}{l}
u_{d h}^{v r} \\
u_{q h}^{v r}
\end{array}\right]=} & T(\Delta \theta)\left[\begin{array}{cc}
L_{d h} & L_{d q h} \\
L_{d q h} & L_{q h}
\end{array}\right] T^{-1}(\Delta \theta) \cdot p\left[\begin{array}{l}
u_{d h}^{v r} \\
u_{q h}^{v r}
\end{array}\right] \\
= & {\left[\begin{array}{c}
L_{s a}-L_{s d} \cos 2 \Delta \theta-L_{d q h} \sin 2 \Delta \theta \\
-L_{s d} \sin 2 \Delta \theta+L_{d q h} \cos 2 \Delta \theta \\
\quad-L_{s d} \sin 2 \Delta \theta+L_{d q h} \cos 2 \Delta \theta \\
\\
\quad L_{s a}+L_{s d} \cos 2 \Delta \theta-L_{d q h} \sin 2 \Delta \theta
\end{array}\right] \cdot p\left[\begin{array}{l}
u_{d h}^{v r} \\
u_{q h}^{v r}
\end{array}\right] }
\end{aligned}
$$

Considering the influence of cross-saturation, the differential of the HF carrier voltage in the virtual synchronous reference frame can be given as

$$
\begin{gathered}
p\left[\begin{array}{l}
u_{d h}^{v r} \\
u_{q h}^{v r}
\end{array}\right] \\
=\left[\begin{array}{cc}
\frac{1}{L_{p}}+\frac{1}{L_{n}} \cos \left(2 \Delta \theta+\theta_{m}\right) & \frac{1}{L_{n}} \sin \left(2 \Delta \theta+\theta_{m}\right) \\
\frac{1}{L_{n}} \sin \left(2 \Delta \theta+\theta_{m}\right) & \frac{1}{L_{p}}-\frac{1}{L_{n}} \cos \left(2 \Delta \theta+\theta_{m}\right)
\end{array}\right] \cdot\left[\begin{array}{c}
u_{d h}^{v r} \\
u_{q h}^{v r}
\end{array}\right] \\
\theta_{m}=\tan ^{-1}\left(\frac{-L_{d q h}}{L_{s d}}\right) \\
L_{p}=\frac{L_{d h} L_{q h}-L_{d q h}^{2}}{L_{s a}}, L_{n}=\frac{L_{d h} L_{q h}-L_{d q h}^{2}}{\sqrt{L_{s d}^{2}+L_{d q h}^{2}}}
\end{gathered}
$$

where $\theta_{m}$ is the cross-saturation angle which is defined in (6a) which is caused by the influence of cross-saturation. $L_{p}$ and $L_{n}$ are referred to the equivalent positive and negative sequence inductances, respectively. $L_{s a}$ and $L_{s d}$ are the average and difference of $d$ - and $q$-axis incremental inductances, which can be defined as

$$
L_{s a}=\frac{L_{q h}+L_{d h}}{2}, \quad L_{s d}=\frac{L_{q h}-L_{d h}}{2}
$$

Injecting a HF pulsating carrier voltage signal (7) into the virtual synchronous reference frame,

$$
\left[\begin{array}{l}
u_{d h}^{v r} \\
u_{q h}^{v r}
\end{array}\right]=U_{c}\left[\begin{array}{c}
\cos \alpha \\
0
\end{array}\right], \alpha=\omega_{c} t+\varphi
$$

where $U_{c}, \omega_{c}$ and $\varphi$ represent the amplitude, angular speed and initial phase angle of the HF injected carrier voltage, respectively. Then, the HF carrier voltages are derived as

$$
\left[\begin{array}{c}
u_{d h}^{v r} \\
u_{q h}^{v r}
\end{array}\right]=\left[\begin{array}{c}
I_{p}+I_{n} \cos \left(2 \Delta \theta+\theta_{m}\right) \\
I_{n} \sin \left(2 \Delta \theta+\theta_{m}\right)
\end{array}\right] \cdot \sin \alpha
$$

where

$$
I_{p}=\frac{U_{c}}{\omega_{c} L_{p}}, \quad I_{n}=\frac{U_{c}}{\omega_{c} L_{n}}
$$

To evaluate the machine saliency information, a simple method for investigating the machine saliency is proposed as shown in Fig 5. With the aid of accurate rotor position information from an incremental encoder, a HF pulsating carrier sinusoidal voltage signal $(7)\left(U_{c}=8 \mathrm{~V}, f_{c}=550 \mathrm{~Hz}\right)$ is injected into the virtual synchronous reference frame $\left(d^{v r}-q^{v r}\right)$, while the machine operates at low speed in the sensored mode, $25 \mathrm{r} /$ min with current about $1.2 \mathrm{~A}$.

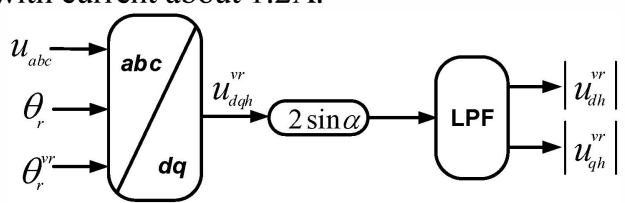

Fig. 5. Principle of machine saliency measurement.

By applying the synchronous detection method [17] and low-pass filter (LPF), the amplitudes of positon-dependent carrier voltages can be obtained based on Type- 1 and Type- 2 as shown in Figs. 6 (a) and (b), and re-written as

$$
\left[\begin{array}{l}
\left|u_{d h}^{v r}\right| \\
\left|u_{q h}^{v r}\right|
\end{array}\right]=L P F\left(\left[\begin{array}{l}
u_{d h}^{v r} \\
u_{q h}^{v r}
\end{array}\right] \cdot 2 \sin \alpha\right)=\left[\begin{array}{c}
I_{p}+I_{n} \cos \left(2 \Delta \theta+\theta_{m}\right) \\
I_{n} \sin \left(2 \Delta \theta+\theta_{m}\right)
\end{array}\right] \text { (9) }
$$

The machine saliency circles are illustrated in Figs. 6 (c) and (d) where the saliency circle indicates the full saliency information. If the circle is bigger, the saliency level is higher. As mentioned earlier, the overall saliency for set A in Type-I and Type-II are the same.

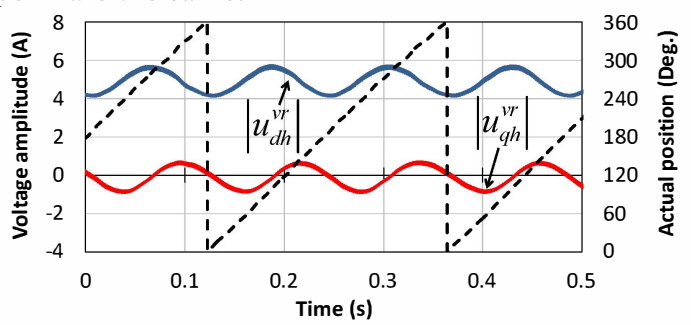

(a) Type-1, set A

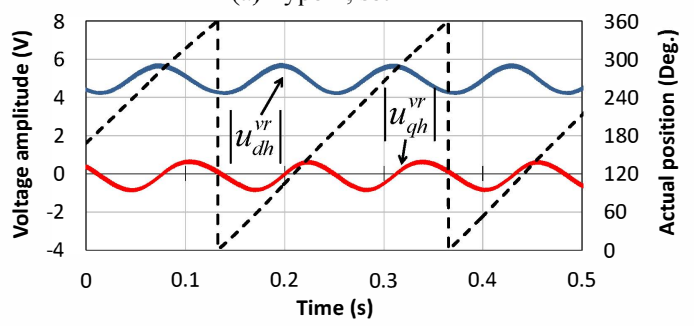

(b) Type-2, set A
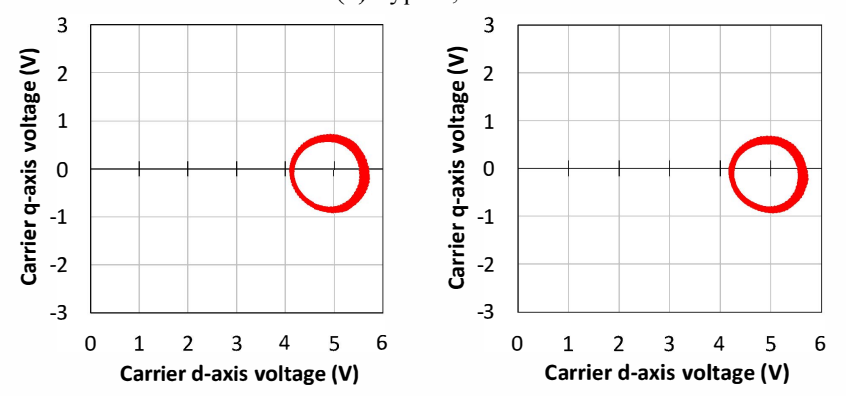

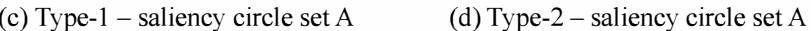
Fig. 6. Measured HF carrier voltage responses based on different types of winding coil connections, $25 \mathrm{r} / \mathrm{min}$. 


\section{Experimental Investigation of Machine Saliency}

Based on the theoretical analysis of machine saliency in the previous section, the machine saliency based on different part of WCCs in Type- 2 needs to be investigated in order to prove the influence of primary saliency or multiple saliencies on the sensorless rotor position estimation.

The same experimental conditions are also applied to the different WCCs based on Type-2. With the aid of mid-tapered winding wires, the $d$ - and $q$-axis carrier voltage responses can be measured from the set A' and set A" at the same time as shown in Figs. 7. Clearly, similar to the principle of carrier current responses, the $d$ - and $q$-axis carrier voltage responses are also modulated as two cycle per electrical position. Moreover, the higher amplitude of carrier voltage response indicates the higher machine saliency level. The experimental results show the saliency level in set A is significantly higher (Fig. 7(a)) than that of the saliency measured from both set A' and set $A$ " as shown in Figs. 7 (b) and (c) due to less winding coils and inductances. Theoretically, set A' have identical saliency level as set A" according the analysis in section II.

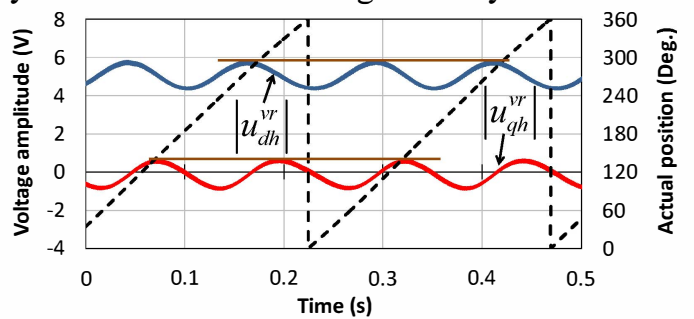

(a) Set A

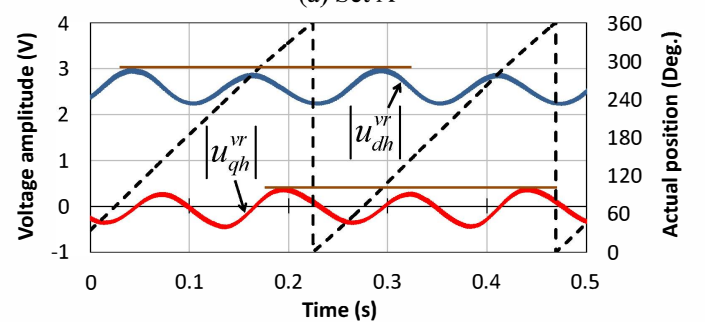

(b) Set A'

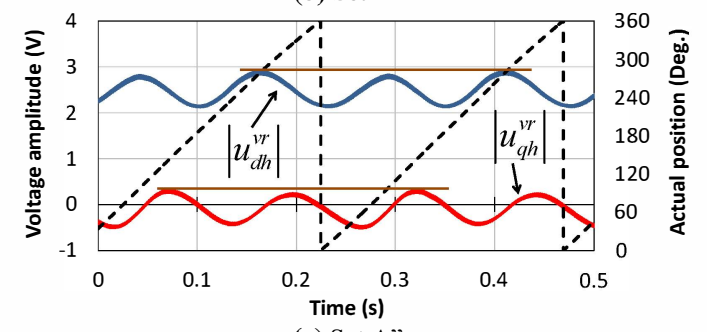

(c) Set A"

Fig. 7. Measured HF amplitude variations of carrier voltage responses based on types of winding coil connections in Type- $2,25 \mathrm{r} / \mathrm{min}$.

Furthermore, the amplitudes of measure $d$ - and $q$-axis carrier voltage responses in set $A$ ' and set $A$ " in Type-2 are non-constant as shown in Figs. 7 (b) and (c), respectively. This non-constant issue is due to the secondary saliency (multiple saliencies effect). However, this issue can be avoided when the winding coils $1,4,7,10$ or $1,7,4,10$ are all connected in series (Type-1 and Type-2).

In Fig. 8, the saliency circles are measured by the mid-tapered winding wire based on the different sequence of WCCs in Type-2. As can be seen from Figs. 8 (b) and (c), set A' has similar saliency level as set A', and both sets are affected by the multiple saliencies, due to which the sensorless rotor position estimation will deteriorate. According to the FFT analyses Fig. 9, Type-1 and Type-2 are the same, and set A' and set A" are almost identical as discussed in section II.

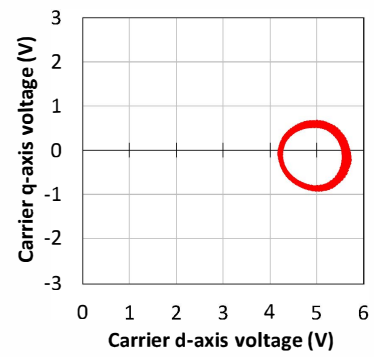

(a) Set A

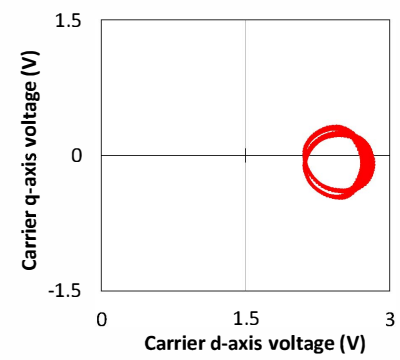

(b) Set A'

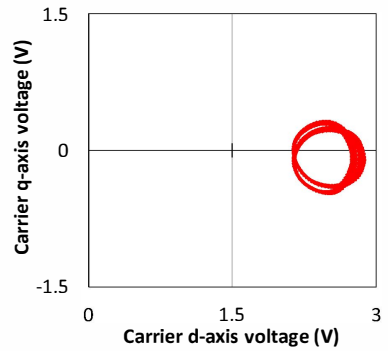

(c) Set A"

Fig. 8. Measured saliency voltage circles based on types of winding coil connections in Type- $2,25 \mathrm{r} / \mathrm{min}$

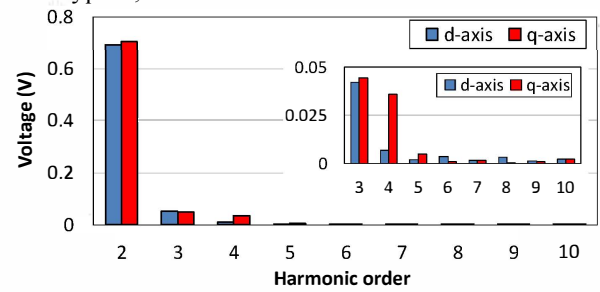

(a) Type-1, Set A

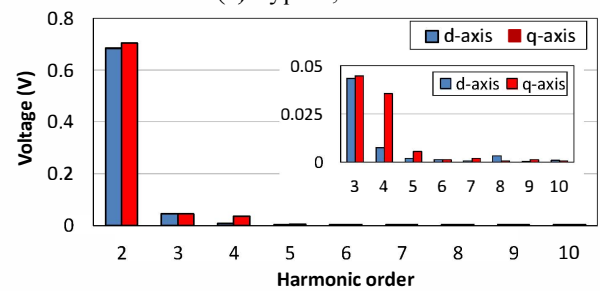

(a) Type-2, set $\mathrm{A}$

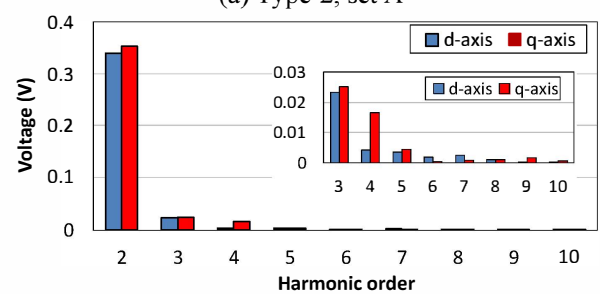

(b) Type-2, set A

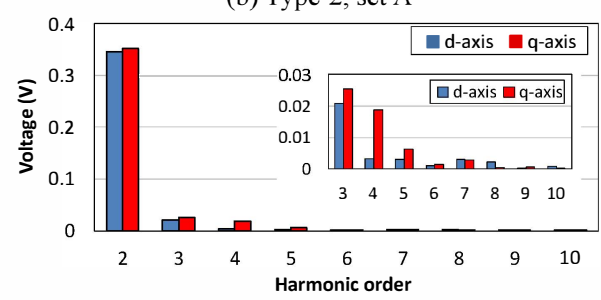

(c) Type-2, set A"

Fig. 9. FFT analysis based on types of winding coil connections in Type-2, 25 $\mathrm{r} / \mathrm{min}$. 


\section{VALIDATION OF SENSORLESS ROTOR POSITON ESTIMATIONS}

A dSPACE platform together with a 12/10 stator/rotor poles prototype SFPM machine shown in Fig. 10 are employed and the machine parameters are listed in Table I. The overall sensorless control scheme based on the HF injection technique is described in Fig. 11. To validate the effectiveness of sensorless rotor position estimation based on the different sequence of WCCs, such as Type-1 and Type-2, as well as the mid-tapered winding wires based HF carrier voltage signal measurement, the HF pulsating carrier voltage injection technique is applied to the prototype SFPM machine. The experimental conditions of injection voltage and frequency for are $U_{c}=8 \mathrm{~V}, f_{c}=550 \mathrm{~Hz}$, which is injected into the estimated synchronous reference frame, and then the rotor position information can be obtained from the carrier voltage response.

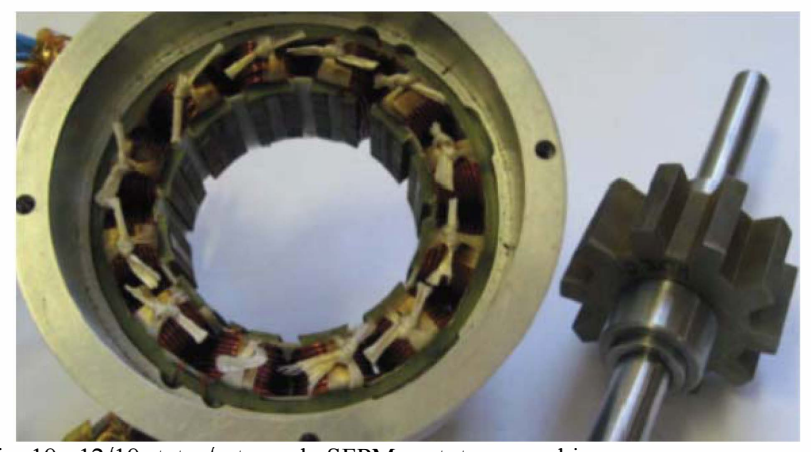

Fig. 10. 12/10 stator/rotor pole SFPM prototype machine

TABLE I

PARAMETERS OF SFPM PROTOTYPE MACHINE

\begin{tabular}{ccc}
\hline \hline Quantity & Value & Unit \\
\hline Rated torque & 2.7 & $\mathrm{Nm}$ \\
Rated speed & 400 & $\mathrm{RPM}$ \\
Stator pole number & 12 & \\
Rotor pole number & 10 & $\mathrm{mH}$ \\
$d$-axis inductance, $L_{\boldsymbol{d}}$ & 0.277 & $\mathrm{mH}$ \\
$q$-axis inductance, $L_{q}$ & 0.339 & \\
\hline \hline
\end{tabular}

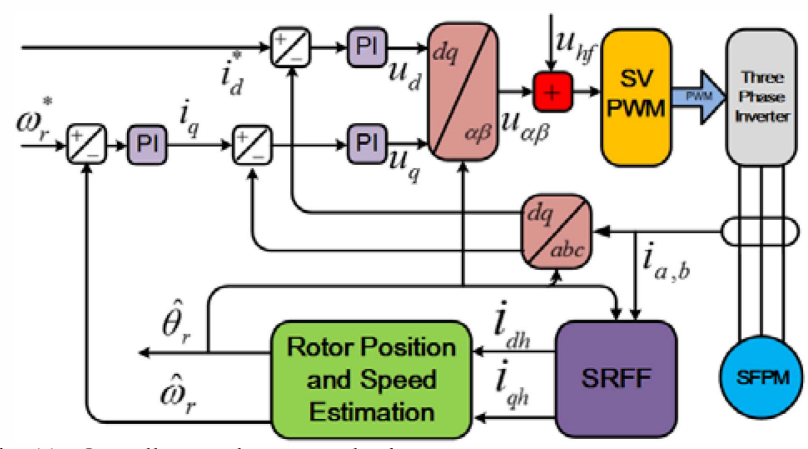

Fig. 11. Overall sensorless control scheme.

\section{A. Single Three-Phase Operation}

Fig. 12 shows the sensorless rotor position estimation at the steady state of constant speed $25 \mathrm{r} / \mathrm{min}$ with current, $2.2 \mathrm{~A}$, by utilizing the mid-tapered winding wires for extracting the HF carrier signals. The experimental results show good sensorless rotor position tracking performance based on the Type- 1 and set A in Type-2, as shown in Figs. 12 (a) and (b), respectively. However, a significant oscillation error will exhibit in the rotor position estimation based on set $A^{\prime}$ and set A" due to the additional harmonics contained in the carrier voltage response caused by the multiple saliencies effect, as shown in Figs. 12 (c) and (d), respectively.

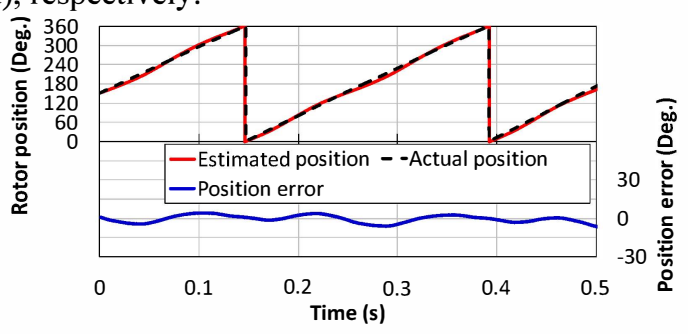

(a) Type-1, set A

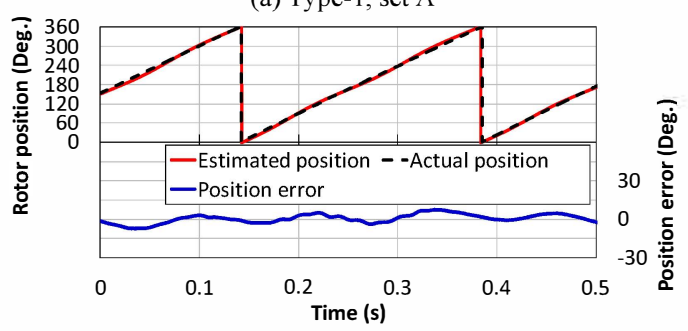

(b) Type-2, set A

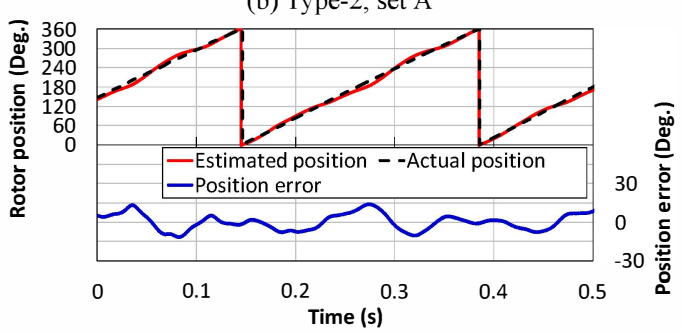

(c) Type-2, set A

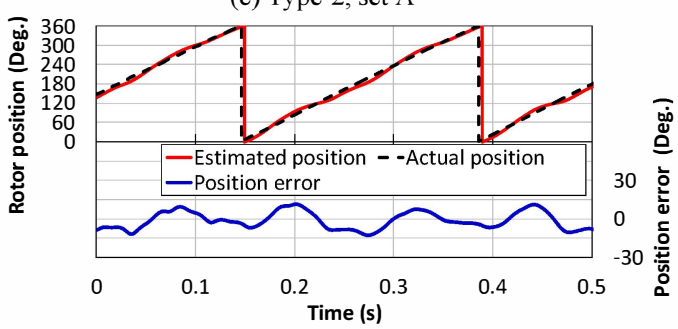

(d) Type-2, set A"

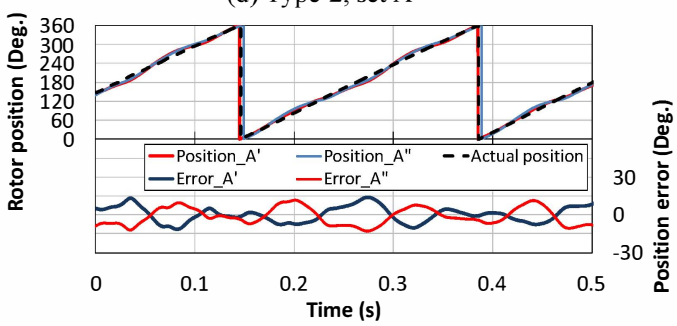

(e) Comparison

Fig. 12. Sensorless rotor position estimations at steady-state, $25 \mathrm{r} / \mathrm{min}$

Additionally, the comparison between set A' and set A" in Type-2 is shown in Fig. 12 (e). As can be seen, the rotor position estimation based on set $A^{\prime}$ and set $A^{\prime \prime}$ have similar performance but the peak error is in the opposite direction with each other, which can be a good feature for the rotor position compensation. According to the saliency analysis shown in Figs. 7 (b) and (c), comparing set A' with set A", there is a phase difference due to HF $d$ - and $q$-axis carrier signals, which can be eliminated by the connection of two sets (A' + A") due to the saliency cancelling effect. Similarly, using the same 
principle to reduce the error in the rotor position estimation, a very simple compensation method is proposed, Fig. 13. When the effect of multiple saliencies can be effectively cancelled, the oscillation error will significantly be reduced. By adding the rotor position estimation errors from both set A' and set A", the average rotor position estimation can be calculated, and then applied to the rotor positon tracking observer. Therefore, high accuracy of rotor positon can be achieved, whilst the oscillation error in the rotor positon estimation can be minimized, as shown in Fig. 14.

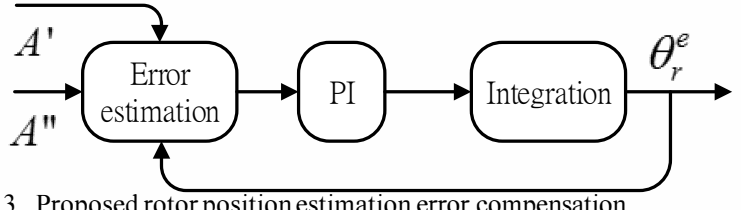

Fig. 13. Proposed rotor position estimation error compensation

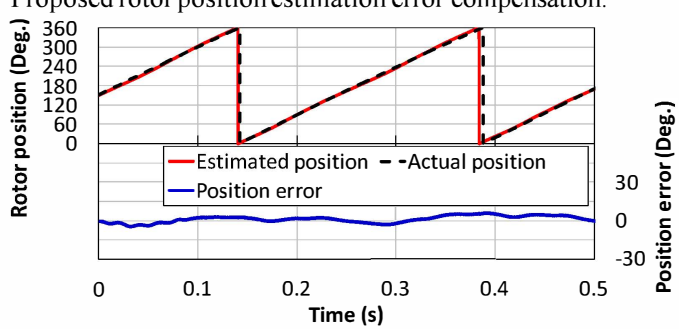

Fig. 14. Improved rotor positon estimation, $25 \mathrm{r} / \mathrm{min}$

\section{B. Dual Three-Phase Operation}

As mentioned in section II, the prototype machine can be operated as the dual three-phase machine, Fig. 15.

Differing from the previous application, the mid-tapered winding wires are not required. Similarly, the same testing conditions are also applied $\left(U_{c}=4 \mathrm{~V}, f_{c}=550 \mathrm{~Hz}\right.$ for each set), the sensorless rotor position estimation at the steady state of constant speed $25 \mathrm{r} / \mathrm{min}$ with current, 2.2A, Fig. 16. However, a significant oscillation error is very obvious in the rotor position estimation based on set 1 and set 2 due to the multiple saliencies effect, as shown in Figs. 16 (a) and (b), respectively, which is the same as the position estimation based on set A' and set A'. The comparison between set 1 and set 2 is shown in Fig. 16 (c). Clearly, the rotor position estimation based on set 1 and set 2 have similar performance as set A' and set A', and the peak errors are in the opposite direction with each other.

Furthermore, by applying the same proposed rotor position estimation error compensation method, Fig. 13, the average rotor position estimation can be re-calculated, and then applied to the rotor positon tracking observer. Therefore, the good sensorless control performance with high accurate rotor position estimation is achieved, as shown in Fig. 17.

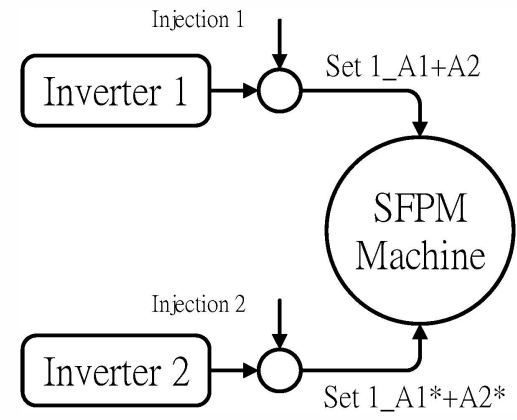

Fig. 15. Control of dual three-phase scheme

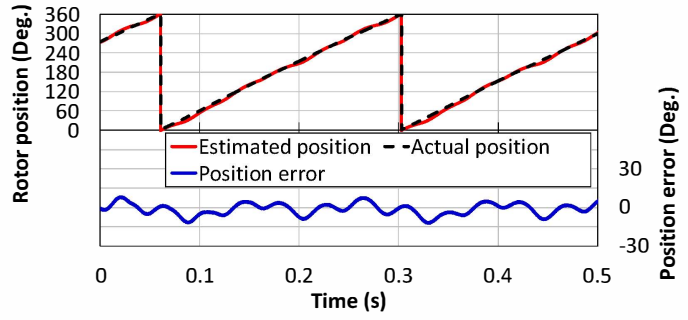

(a) A1 and A2, set 1

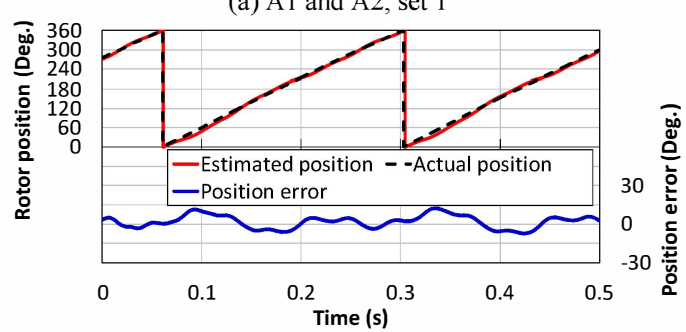

(b) $\mathrm{A} 1 *$ and $\mathrm{A} 2 *$, set 2

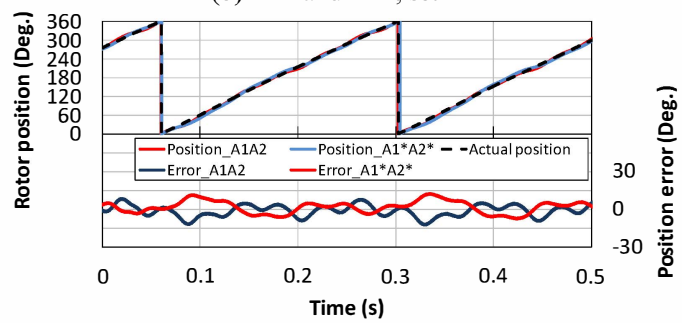

(c) Comparison

Fig. 16. Sensorless rotor position estimations at steady-state, $25 \mathrm{r} / \mathrm{min}$.

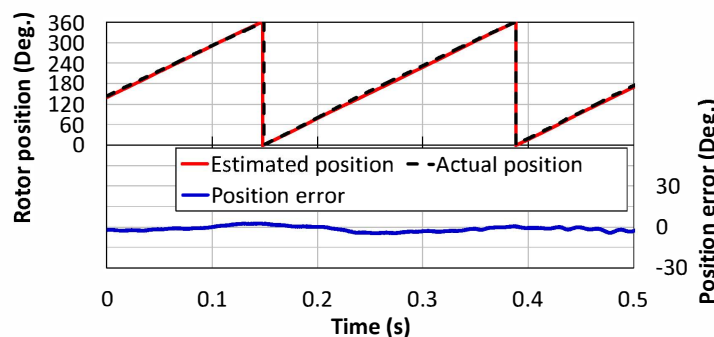

Fig. 17. Improved rotor positon estimation, $25 \mathrm{r} / \mathrm{min}$.

\section{CONCLUSION}

In this paper, the sensorless rotor position estimations based on the different sequence of WCCs (Type-1 and Type-2) have experimentally investigated as well as the different WCCs in Type-2. Moreover, with the aid of mid-tapered winding wires, the machine saliency can be measured from set A' and set A" separately, of which the saliency level for set A' and set A" are similar but with phase shift, whilst the multiple saliencies are contained.

Since the secondary saliency exists in set A' and set A" based on Type-2 WCC which will deteriorate the accuracy of the rotor position estimation. The proposed method can successfully cancel the additional harmonics in the HF carrier signals. Hence, better performance and more accurate rotor position estimation are achieved. Also, the proposed error cancellation method is applicable to dual three-phase SFPM machines, as confirmed by the experiments. 


\section{REFERENCE}

[1] P.L. Jansen and R.D. Lorenz, "Transducerless position and velocity estimation in induction and salient AC machines," IEEE Trans. Ind. Appl., vol. 31, no. 2, pp. 240-247, 1995.

[2] M.W Degner and R.D Lorenz, "Using multiple saliencies for the estimation of flux, position, and velocity in AC machines," IEEE Trans. Ind. Appl., vol. 34, no. 5, pp. 1097-1104, 1998

[3] D. Raca, P. Garcia, D. Reigosa, F. Briz, and R.D. Lorenz, "A comparative analysis of pulsating vs. rotating vector carrier signal injection-based sensorless control," Proc. Of APEC2008, Feb. 2008, pp. 879-885.

[4] Y. Li, Z.Q. Zhu, D. Howe, C.M. Bingham, "Modeling of cross-coupling magnetic saturation in signal-injection-based sensorless control of permanent-magnet brushless AC motors," IEEE Trans. Magnet., vol. 43, no. 6, pp.2552-2554, 2007.

[5] N. Bianchi and S. Bolognami, "Influence of rotor geometry of an IPM motor on sensorless control feasibility," IEEE Trans. Ind. Appl., vol. 43, no. 1, pp. 87-96, 2007

[6] L.M. Gong, and Z.Q. Zhu, "A novel method for compensating inverter nonlinearity effects in carrier signal injection-based sensorless control from positive-sequence carrier current distortion," IEEE Trans. Ind. Appl., vol. 47, no. 3, pp. 1283 - 1292, 2011

[7] Z. Q. Zhu, Y. Pang, D. Howe, S. Iwasaki, R. Deodhar, and A. Pride, "Analysis of electromagnetic performance of flux-switching permanent magnet machines by nonlinear adaptive lumped parameter magnetic circuit model," IEEE Trans. on Magn., vol. 41, no. 11, pp.4277-4287, Nov. 2005

[8] E. Hoang, A.H. Ben-Ahmed, and J. Lucidarme, "Switching flux permanent magnet poly phased synchronous machines," Proc. 7th Eur. Conf. Power Electronics and Applications, vol. 3, pp. 903-908, 1997.

[9] Wei Hua, Ming Cheng, Z.Q. Zhu, and D. Howe, "Analysis and optimization of back-EMF waveform of a flux-switching permanen magnet motor," IEEE Trans. Energy Conversion, vol. 23, no. 3, pp 727-733, Sep. 2008 